\title{
Tamanho Ótimo Amostral e Análise Fatorial e Correlacional do Desempenho de Indivíduos sob a Influência de Plataformas Computacionais de Apoio ao Ensino
}

\author{
Taffarel Brant-Ribeiro ${ }^{1}$ e Renan G. Cattelan ${ }^{2}$ \\ ${ }^{1}$ Instituto Federal de Educação, Ciência e Tecnologia do Sul de Minas Gerais \\ Passos / MG - Brasil \\ ${ }^{2}$ Faculdade de Computação - Universidade Federal de Uberlândia \\ Uberlândia / MG - Brasil \\ brant.ribeiro@ifsuldeminas.edu.br, renan@ufu.br
}

\begin{abstract}
In literature related to the area of Informatics in Education, many papers which analyze new technological proposals are only aimed at observing improvements that the usage of tools can cause. Such researches do not analyze whether the sample size is robust to ensure reliability of results or the tool enhancement tends to maintain some influence over students' performance. Based on this, our work determined optimal sample sizes for the performance analysis of students with/without contact with teaching support technologies. An Experiments Manager was also developed to organize the functionalities visibility of a Ubiquitous Learning Environment (ULE) and, using this Experiments Manager, a Factorial Analysis of Variance and a Correlation Analysis were performed. It was observed that undergraduate students' performance was influenced by the interaction between ULE functionalities and the courses taken by students. In graduate classes, there was no significant difference in students performance between ULE levels of usage, nor the occurrence of correlations that indicated something similar to what happened with undergraduates, although there have also been increases in students' performance at this academic level.
\end{abstract}

Resumo. Na literatura relacionada à área de Informática na Educação, muitos trabalhos que analisam novas propostas tecnologicas têm como objetivo apenas observar as melhorias que o uso das ferramentas pode ocasionar. Tais pesquisas não analisam se o tamanho amostral é robusto para garantir confiabilidade nos resultados ou se o aprimoramento das ferramentas tende a manter alguma influência sobre o desempenho discente. Com base nisso, este trabalho determinou tamanhos ótimos amostrais para a análise do desempenho de turmas com e sem contato com plataformas educacionais. Também foi desenvolvido um Gerenciador de Experimentos para se organizar a visibilidade das funcionalidades de um Ambiente Educacional Ubíquo (AEU) e, empregando-se esse gerenciador, foram realizadas Análises de Variância Fatorial e Correlacional. Percebeu-se que o desempenho dos alunos de graduação foi influenciado pela interação entre as funcionalidades do AEU e as disciplinas cursadas pelos estudantes. Na pós-graduação, não se observou diferença significativa no desempenho discente entre os níveis de uso do AEU, nem a ocorrência de correlações que indicassem algo similar ao que ocorreu na graduação, embora também tenham ocorrido acréscimos no desempenho obtido pelos estudantes neste nível acadêmico. 
VIII Congresso Brasileiro de Informática na Educação (CBIE 2019)

Anais dos Workshops do VIII Congresso Brasileiro de Informática na Educação (WCBIE 2019)

\section{Introdução}

O uso de dispositivos tecnológicos propagou-se de modo intenso na sociedade, transformando os computadores em elementos onipresentes que se entrelaçaram por completo ao modo de vida das pessoas [Internet World Stats 2018]. No âmbito acadêmico, o emprego de ferramentas que têm como intuito apoiar o processo de ensino/aprendizagem tornou-se uma atividade habitual, contribuindo para a validação de teorias pedagógicas ao possibilitar o desenvolvimento de novas plataformas de apoio ao ensino que empregam sistemas de hardware e software criados especificamente para esta finalidade [Knight et al. 2015, Gasevic et al. 2017, Cai 2018].

$\mathrm{Na}$ literatura, trabalhos sobre desempenho e adaptabilidade de usuários em contato com plataformas educacionais são frequentemente observados e abrem margem para diversas análises, como, por exemplo, a verificação de ganhos para estudantes que fazem uso cotidiano de ferramentas acadêmicas [Mouri et al. 2015]. No entanto, a maioria dessas pesquisas preocupa-se apenas em fazer comparações entre usuários, expondo que existem aumentos significativos de desempenho entre grupos analisados [Dickson et al. 2012, Mouri et al. 2015], mas não dedicando-se em averiguar inicialmente se o tamanho das amostras utilizadas é robusto o suficiente para representar de modo fiel as populações de indivíduos das quais são retiradas.

Com base nisso, este trabalho almejou inicialmente criar um módulo de gerenciamento de experimentos capaz de organizar o nível de visibilidade de funcionalidades experimentais e refinamentos presentes em Ambientes Educacionais Ubíquos (AEUs) e fornecer subsídios para a realização de pesquisas nesta vertente. Como estudo de caso, tal módulo foi implementado em um AEU denominado Classroom eXperience (CX) [Brant-Ribeiro and Cattelan 2016, Brant-Ribeiro and Cattelan 2018], que se trata de uma plataforma educacional desenvolvida para se registrar fluxos de conteúdo apresentados em sala de aula e disponibilizá-los posteriormente para alunos e professores da Faculdade de Computação da Universidade Federal de Uberlândia (FACOM/UFU).

Em paralelo ao desenvolvimento do Gerenciador de Experimentos, esta pesquisa também objetivou determinar um tamanho ótimo amostral para a análise do desempenho de estudantes de graduação e pós-graduação em contato com plataformas educacionais. Além disso, enxergou-se também a necessidade de se determinar um tamanho ótimo amostral para analisar alunos de graduação e pós-graduação que não estão em contato com tais tecnologias, de modo a ser possível traçar um paralelo entre os resultados obtidos e se recomendar uma estimativa capaz de compreender ambas as circunstâncias.

Em seguida, foram realizadas duas investigações: uma Análise de Variância (ANOVA) Fatorial 4x5 e uma Correlação de Spearman. A ANOVA Fatorial foi realizada para se atestar a existência de diferenças significativas no desempenho acadêmico de alunos em contato com o AEU à medida que o mesmo foi aprimorado por meio da inclusão de novas funcionalidades que foram disponibilizadas para os discentes durante os semestres letivos. Já o estudo que empregou a Correlação de Spearman foi realizado para averiguar possíveis relações entre o desempenho acadêmico obtido pelos estudantes e seus fluxos de interação com o CX. Desta maneira, foi possível obter indícios acerca do processo de evolução de uma plataforma de apoio ao ensino e o impacto que ela causou progressivamente no desempenho de estudantes em um ambiente acadêmico real. 
VIII Congresso Brasileiro de Informática na Educação (CBIE 2019)

Anais dos Workshops do VIII Congresso Brasileiro de Informática na Educação (WCBIE 2019)

\section{Gerenciador de Experimentos}

Para a realização desta pesquisa, foi desenvolvido um Gerenciador de Experimentos com características generalistas e capaz de ser empregado para realizar o controle da visibilidade de módulos e funcionalidades em plataformas de apoio ao ensino, tais como AEUs. $\mathrm{O}$ modelo aqui proposto compreendeu um mecanismo que realiza o controle de quais funcionalidades devem ser exibidas para usuários, de modo a ser possível habilitá-las em tratamentos diferentes e para grupos distintos de indivíduos (Figura 1).

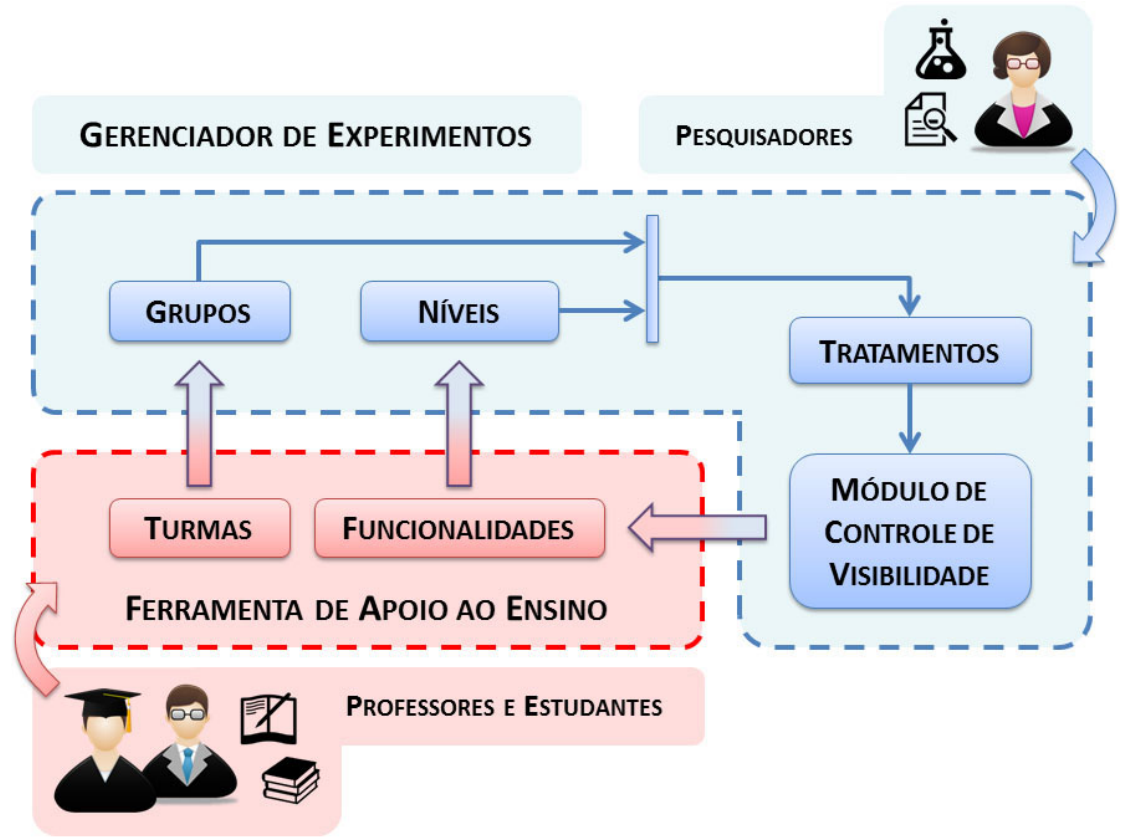

\section{Figura 1. Estrutura do Gerenciador de Experimentos e interface de comunicação com ferramentas de apoio ao ensino (Fonte: [Brant-Ribeiro and Cattelan 2018]).}

O Gerenciador de Experimentos (realçado em azul na Figura 1) compreende um módulo de organização de dados provenientes de ferramentas acadêmicas. No âmbito de plataformas educacionais, as turmas que possuem estudantes matriculados em disciplinas cadastradas nessas ferramentas são caracterizadas como grupos (ou amostras) experimentais a serem utilizados pelo gerenciador. Além disso, cada funcionalidade presente na plataforma fornece dados referentes aos níveis de acesso a essas funcionalidades, visto que as mesmas podem ser habilitadas ou desabilitadas para cada usuário ou, também, para conjuntos de usuários que fazem parte de uma mesma turma.

Empregando-se os dados dos grupos e dos níveis de acesso às funcionalidades, os pesquisadores podem montar tratamentos, que são organizações dos níveis de visibilidade das funcionalidades que serão habilitadas para cada grupo de estudantes. Esses tratamentos são organizados utilizando-se um módulo de controle de visibilidade das funcionalidades nas plataformas, capaz de regular e restringir o que cada usuário poderá acessar em cada uma das turmas que fizer parte. Por se tratar de um elemento capaz de realizar o controle de funcionalidades e módulos que compõem as ferramentas de apoio ao ensino, apenas pesquisadores devem ter acesso ao módulo de gerenciamento. Por causa disso, o mesmo mostra-se invisível para professores e alunos que empregam tais ferramentas no decorrer dos semestres letivos. 
VIII Congresso Brasileiro de Informática na Educação (CBIE 2019)

Anais dos Workshops do VIII Congresso Brasileiro de Informática na Educação (WCBIE 2019)

\section{Cálculo de Tamanho Ótimo Amostral}

Para a realização desta etapa da pesquisa, foram utilizados dados provenientes de dez turmas que cursaram disciplinas da grade curricular dos cursos de graduação e pósgraduação da FACOM, totalizando 365 observações coletadas no decorrer de três anos letivos (de 2013 a 2015). Dessas, cinco não tiveram qualquer contato com a plataforma de apoio ao ensino CX, ao passo que as demais utilizaram o AEU no decorrer dos semestres letivos. Objetivando compreender estudantes tanto do curso de graduação em Sistemas de Informação quanto dos cursos de graduação e pós-graduação em Ciência da Computação, foram selecionadas disciplinas que todos os cursos possuem em suas respectivas grades curriculares, de modo a propositalmente haver amostras mistas com quantidades diversificadas de estudantes de cada curso.

As dez amostras selecionadas foram compostas por $N$ observações da variável sob análise. Tendo em vista que cada amostra compreendeu uma turma de alunos matriculados numa respectiva disciplina, o tamanho das amostras não se manteve fixo e apresentou variações entre 26 e 44 estudantes. Como a variável estudada nesta pesquisa foi o desempenho acadêmico dos alunos, as notas dos estudantes foram coletadas ao final de cada semestre letivo e, para cada uma das amostras resultantes, foram realizadas combinações aleatorizadas (reamostragens) das observações para formar subamostras de diversos tamanhos a partir da amostra original.

Para a realização do processo de reamostragem, o método Bootstrapping foi empregado [Efron 1979]. Deste modo, para cada subamostra de tamanho $n$ originada de uma amostra de tamanho $N$, foram realizadas 2000 reamostragens para todo $n \leq N$. Em seguida, para cada um dos conjuntos de 2000 subamostras de mesmo tamanho resultantes, realizou-se o cálculo da Média $(\bar{x})$, Variância $\left(s^{2}\right)$, Desvio Padrão $(s)$, Coeficiente de Variação (CV), além de um Intervalo de Confiança (IC) de 95\% para essas medidas. Para o cálculo do tamanho ótimo amostral, empregou-se o Método de Máxima Curvatura Modificado (MMCM) [Lessman and Atkins 1963, Meier and Lessman 1971]. Deste modo, inicialmente representou-se a relação entre cada CV obtido e seu conjunto de 2000 subamostras de mesmo tamanho empregando-se a equação de regressão de potência do MMCM e, após esta etapa, realizou-se o cálculo do Coeficiente de Determinação $\left(R^{2}\right)$ [Ozer 1985] para cada uma das turmas.

Por fim, representou-se a relação entre os CVs calculados e os tamanhos das subamostras de maneira gráfica em eixos cartesianos, nos quais o eixo $y$ representou o CV e o eixo $x$ retratou o intervalo dos tamanhos de amostra. Após estabelecer as estimativas dos $\mathrm{CV}$ s de todas as subamostras de cada turma analisada, calculou-se o valor referente ao ponto da abcissa no qual ocorre a máxima curvatura do modelo utilizado, correspondente ao tamanho ótimo de amostra, denominado $X_{M C}$.

\subsection{Resultados e Discussões}

A Figura 2 apresenta os dez eixos cartesianos correspondentes às amostras analisadas, sendo os cinco dispostos na primeira coluna (gráficos de $\mathrm{A}$ a E) referentes às turmas que não tiveram contato com o $\mathrm{CX}$ e os demais eixos (gráficos de $\mathrm{F}$ a $\mathrm{J}$ ) relativos às turmas que empregaram o sistema durante o semestre letivo. Os CVs exatos de cada tamanho de subamostra foram representados em seus respectivos eixos junto à equação de regressão de potência que indicou a tendência dos dados obtidos. Também calcularam-se os limites 
VIII Congresso Brasileiro de Informática na Educação (CBIE 2019)

Anais dos Workshops do VIII Congresso Brasileiro de Informática na Educação (WCBIE 2019)
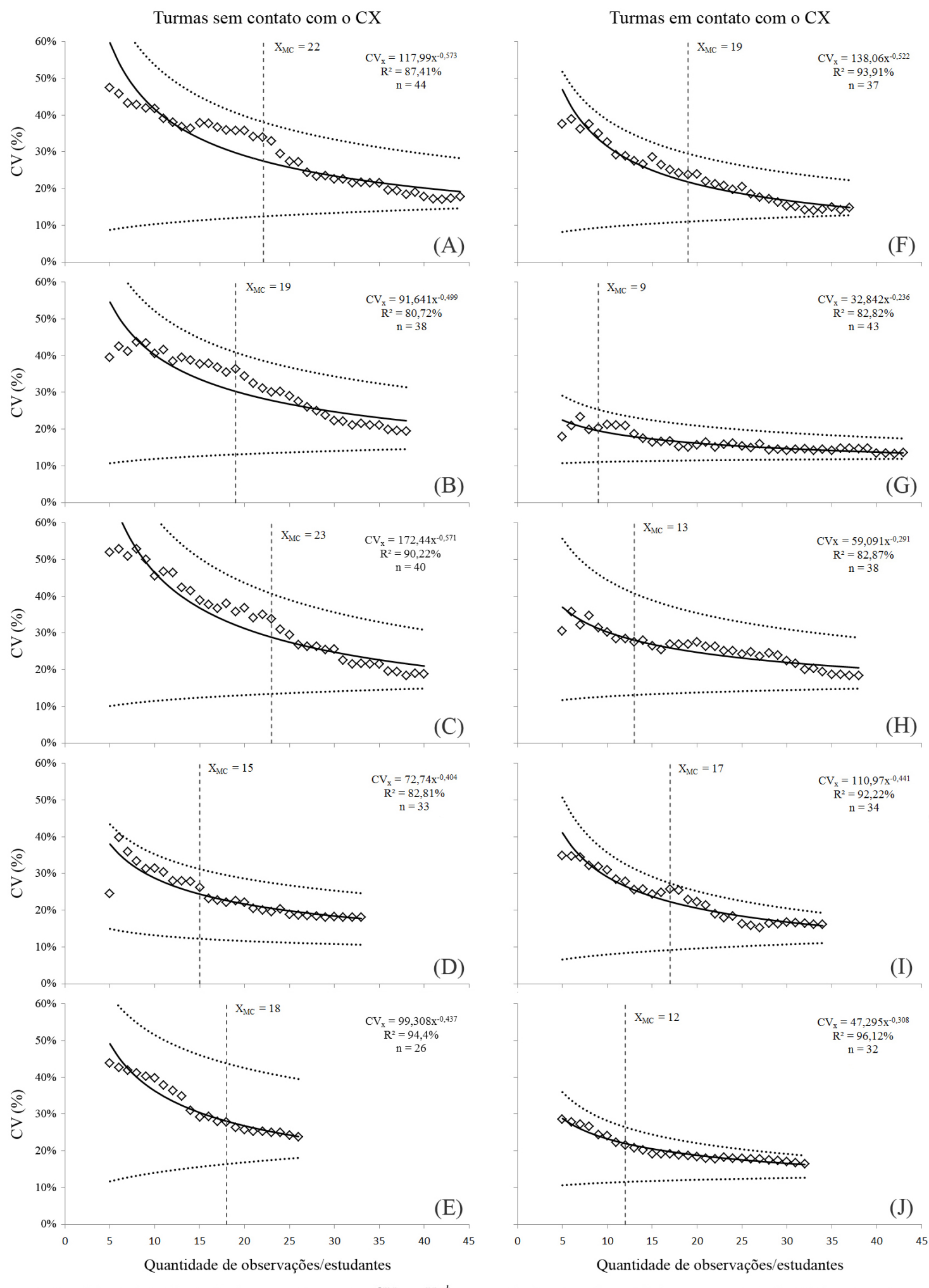

$\diamond$ Coeficiente de Variação $(\mathrm{CV}) \quad-$ Regressão $C V_{x}=a X^{-b}$

... Limites Superior e Inferior ‥- Ponto de Máxima Curvatura

Figura 2. Relações entre os CVs e os tamanhos de subamostras para a variável desempenho dos estudantes em dez turmas de cursos da área computacional (Fonte: [Brant-Ribeiro and Cattelan 2016]). 
VIII Congresso Brasileiro de Informática na Educação (CBIE 2019)

Anais dos Workshops do VIII Congresso Brasileiro de Informática na Educação (WCBIE 2019)

superiores e inferiores dos CVs, optando-se por representá-los já aplicados ao modelo, de modo a demonstrar os pontos máximos e mínimos que os CVs seriam capazes de alcançar em cada tamanho de subamostra sob um IC de $95 \%$.

A partir da análise das dez turmas utilizadas, foi possível perceber que o valor do $\mathrm{CV}$ diminuiu ao passo que o tamanho das subamostras aumentou. Por meio do MMCM, o tamanho ótimo de amostra obtido entre as cinco turmas que não tiveram contato com o CX variou entre 15 e 22 estudantes, ao passo que nas turmas que empregaram o sistema durante os semestres letivos a estimativa foi de 9 a 19 alunos.

Com base nesses resultados, torna-se possível compreender que as turmas que utilizaram o AEU no decorrer dos semestres letivos tenderam a apresentar pontos de máxima curvatura inferiores aos obtidos naquelas que não tiveram contato com a tecnologia. Isso demonstra que quantidades de observações menores que as originalmente existentes nas amostras já teriam sido suficientes para representar o comportamento da variável sob análise. Além disso, como entre as turmas que utilizaram o CX o nível médio de variabilidade das notas foi ainda menor que naquelas que não utilizaram o sistema, é possível ratificar a hipótese de que maiores índices de nivelamento entre o desempenho dos alunos podem ocorrer em razão dos mesmos estudarem os conteúdos das disciplinas a partir de uma fonte em comum.

\section{Análise Fatorial}

Para a realização da Análise Fatorial, foram utilizados dados provenientes de vinte turmas que cursaram disciplinas da grade curricular dos cursos de graduação em Sistemas de Informação e graduação e pós-graduação em Ciência da Computação da FACOM, totalizando 501 observações. A variável analisada foi o desempenho obtido pelos estudantes em disciplinas cursadas durante seis anos letivos (de 2012 a 2017) e, para isso, as notas alcançadas pelos discentes foram coletadas ao final de cada semestre letivo. De modo a assegurar a confiabilidade desses dados, objetivou-se que os mesmos professores permanecessem lecionando suas respectivas disciplinas durante os semestres analisados, sendo também orientados sobre a necessidade de empregarem uma sequência similar de exposição de conteúdos em suas aulas, objetos de avaliação semelhantes e que os mesmos fossem aplicados em datas proporcionalmente aproximadas.

Inicialmente, as observações coletadas (notas dos estudantes) foram submetidas ao teste de Shapiro-Wilk para verificar se os resíduos da variável observada seguiam a distribuição normal e ao teste de Levene para averiguar se as amostras possuíam variâncias homogêneas. Posteriormente, realizou-se uma Análise de Variância Fatorial 4x5 para se atestar a existência de diferenças significativas entre os níveis de dois fatores investigados. O primeiro fator, denominado "Uso do CX", caracterizou-se como o modo com que o CX foi empregado pelos estudantes e possuiu quatro níveis distintos: 1) CX Ausente, 2) Uso isolado do CX, 3) Uso do CX + Módulo Social (MS) e 4) Uso do CX + MS + Gamificação. O segundo fator, intitulado "Disciplinas", compreendeu todas as disciplinas que foram cursadas pelos alunos empregando os níveis de uso do CX supracitados e possuiu cinco níveis: Lógica Matemática, Otimização, Arquitetura e Organização de Computadores e Interação Humano-Computador (tanto na Graduação quanto na Pós-graduação). 
VIII Congresso Brasileiro de Informática na Educação (CBIE 2019)

Anais dos Workshops do VIII Congresso Brasileiro de Informática na Educação (WCBIE 2019)

\subsection{Resultados e Discussões}

A realização dos testes de Shapiro-Wilk, Levene e $F$ de Snedecor possibilitou atestar a existência de diferenças significativas entre os níveis dos fatores "Uso do CX" e "Disciplinas". Como resultados, na Tabela 1 é possível observar que a ANOVA apresentou efeito significativo em três circunstâncias distintas: o uso do CX (F1), as disciplinas cursadas (F2) e também para a existência de uma interação entre os dois fatores (F1 x F2).

Tabela 1. Análise de Variância Fatorial do desempenho discente com relação ao uso do CX em disciplinas de graduação e pós-graduação em cursos da área computacional (Fonte: [Brant-Ribeiro and Cattelan 2018]).

\begin{tabular}{cccc}
\hline Fatores de Variação $^{1}$ & Graus de Liberdade & Quadrado Médio & $F(P)$ \\
\hline Uso do CX (F1) & 3 & 3263,554 & $24,044(<0,01)$ \\
Disciplinas (F2) & 4 & 3971,876 & $29,263(<0,01)$ \\
Interação (F1 x F2) & 12 & 648,836 & $4,780(<0,01)$ \\
Resíduo & 481 & 135,730 & \\
\hline$C V(\%) ; W(P) ; F^{\prime}(P)$ & 14,$92 ; 0,0961(<0,01) ; 3,110(0,062)$ \\
\hline
\end{tabular}

${ }^{1} F(P)$ : Estatística e Probabilidade do teste $F$ de Snedecor; Probabilidades menores que 0,05 indicam a existência de ao menos um tratamento (nível) diferente dos demais; $C V$ : Coeficiente de Variação; $W(P)$ e $F^{\prime}(P)$ : Estatísticas dos testes de Shapiro-Wilk e Levene; Probabilidades maiores que 0,05 indicam resíduos com distribuição normal e variâncias homogêneas, respectivamente.

Devido a isso, o maior impacto deste resultado incide sobre a característica de interação entre o uso do AEU e as disciplinas cursadas pelos estudantes, ou seja, o desempenho dos alunos mostrou-se de fato influenciado tanto pelo nível em que a ferramenta foi utilizada quando pelo tipo de disciplina cursada. Tal resultado permite deduzir que a natureza das disciplinas sob análise (sejam elas teóricas ou práticas, por exemplo) possui impacto sobre o desempenho obtido pelos estudantes e mostra-se como um fator que também deve ser levado em consideração ao se realizar análises que objetivam observar o impacto do uso de plataformas de apoio ao ensino.

\section{Análise Correlacional}

Para a realização da Análise Correlacional, foram utilizados dados advindos de quinze turmas que cursaram disciplinas da grade curricular dos cursos de graduação em Sistemas de Informação e graduação e pós-graduação em Ciência da Computação da FACOM, totalizando 378 observações. Tais dados compreenderam um conjunto amostral semelhante ao empregado para a realização da ANOVA, no entanto, nesta etapa foram desconsideradas as turmas que não tiveram contato com o CX durante os períodos letivos. Enxergou-se essa necessidade devido ao objetivo desta análise ter sido observar a relação entre a nota alcançada pelos estudantes e as interações que os mesmos tiveram com a plataforma CX, sendo, portanto, dispensável o emprego de turmas que não utilizaram a ferramenta.

A principal variável analisada foi o desempenho acadêmico obtido pelos estudantes em disciplinas cursadas durante seis anos letivos (de 2012 a 2017) e, para isso, suas notas foram coletadas ao final de cada semestre letivo. As disciplinas empregadas nesta análise foram: Lógica Matemática, Otimização, Arquitetura e Organização de 
VIII Congresso Brasileiro de Informática na Educação (CBIE 2019)

Anais dos Workshops do VIII Congresso Brasileiro de Informática na Educação (WCBIE 2019)

Computações e Interação Humano-Computador (Graduação e Pós-Graduação). Para cada disciplina, foram coletadas as notas de turmas distintas que tiveram três níveis de contato com a plataforma CX: A) Uso isolado do CX, B) Uso do CX junto ao Módulo Social (MS) e C) Uso do CX junto ao MS e à Gamificação.

Deste modo, realizou-se uma análise correlacional entre o desempenho discente e a quantidade de logins realizados na plataforma CX. Para esta finalidade, os níveis de uso do CX foram organizados em quatro grupos: A) Uso isolado do CX, B) Uso do CX junto ao MS, C) Uso do CX junto ao MS e à Gamificação e D) Agrupamento desses três níveis para investigar se a relação entre o desempenho dos alunos e a quantidade de logins realizados mostrava-se como um fenômeno concentrado ou espalhado entre as diferentes turmas que cursaram a mesma disciplina.

\subsection{Resultados e Discussões}

A Correlação de Spearman foi empregada para averiguar se os estudantes que obtiveram as maiores notas das quinze turmas analisadas também foram aqueles que realizaram a maior quantidade de logins na plataforma durante os semestres letivos. Os resultados obtidos podem ser observados na Tabela 2.

Tabela 2. Correlação de Spearman entre o desempenho acadêmico obtido pelos estudantes e seus respectivos logins na plataforma CX.

\begin{tabular}{cccccc}
\hline \multirow{2}{*}{ Uso do CX } & \multicolumn{5}{c}{ Disciplinas } \\
\cline { 2 - 6 } & LM & Otimização & AOC & IHC (Grad) & IHC (Pós) \\
\hline A & $0,187 b$ & $0,228 b$ & 0,074 & 0,068 & 0,035 \\
B & $0,241 b$ & $0,193 b$ & $0,232 b$ & $0,204 b$ & 0,069 \\
C & $0,326 m$ & $0,447 m$ & $0,269 b$ & $0,127 b$ & $0,287 b$ \\
D & 0,072 & 0,095 & 0,051 & 0,024 & 0,016 \\
\hline
\end{tabular}

${ }^{1} \rho$ : Coeficiente da Correlação de Spearman; $b$ : Correlação baixa; $m$ : Correlação moderada; LM: Lógica Matemática; AOC: Arquitetura e Organização de Computadores; IHC: Interação Humano-Computador; Grad: Turmas de graduação; Pós: Turmas de pós-graduação; A: Uso isolado do CX; B: Uso do CX junto ao Módulo Social; C: Uso do CX junto aos Módulos Social e de Gamificação; D: Agrupamento dos três níveis de uso do CX numa única amostra.

A partir da análise da Tabela 2, é possível perceber que não houve nenhuma correlação para as disciplinas na circunstância em que as amostras tiveram as observações agrupadas (nível D). Isso indica que a relação entre as variáveis realmente possui efeitos mais concentrados e que os diferentes módulos acrescentados ao AEU ocasionaram quantidades diversificadas de acessos à plataforma. Tal indício pode ser confirmado ao se analisar que em todas as turmas que fizeram uso do AEU no nível $\mathrm{C}$ houve algum tipo de correlação, ao passo que entre as amostras que empregaram o sistema sem o acréscimo de módulos (nível A), apenas duas turmas apresentaram correlação baixa.

A disciplina IHC (Pós-graduação) apresentou apenas uma correlação baixa. A ocorrência reduzida de correlações neste nível de ensino pode ser explicada devido aos estudantes de pós-graduação já possuírem suas próprias técnicas de estudo e serem naturalmente esforçados, o que faz com que seus desempenhos já sejam elevados com ou 
VIII Congresso Brasileiro de Informática na Educação (CBIE 2019)

Anais dos Workshops do VIII Congresso Brasileiro de Informática na Educação (WCBIE 2019)

sem a utilização de plataformas de apoio ao ensino. Infere-se, portanto, que a utilização de propostas tecnológicas na pós-graduação acaba se mostrando apenas como mais uma possibilidade dentre as existentes, diluindo sua importância e eficácia de uso.

Como ocorreram correlações baixas e moderadas em todas as disciplinas de graduação aqui analisadas, seja em menor escala - como em AOC e IHC (Graduação) - ou na maior parte dos casos - como em LM e Otimização -, é possível inferir que os estudantes de cursos de graduação que obtiveram os desempenhos mais elevados também foram aqueles que mais empregaram o $\mathrm{CX}$ no decorrer dos semestres letivos e se beneficiaram do uso da ferramenta. O uso de uma plataforma de apoio ao ensino, neste caso, evidencia-se como uma abordagem relevante para auxiliar os estudantes durante os processos de aprendizagem que ocorrem em cursos de graduação.

\section{Conclusões}

Esta pesquisa determinou um tamanho ótimo amostral de 25 alunos para a análise do desempenho acadêmico em turmas que não utilizam tecnologias educacionais no cotidiano e de 20 estudantes para turmas que possuem contato com tecnologias educacionais durante os semestres letivos. Percebeu-se que a diminuição do CV das amostras não apresentou um comportamento proporcional à elevação da quantidade de observações, havendo uma redução drástica na variabilidade quando as amostras adquiriram tamanhos aceitáveis, mas que tendeu à estabilidade ao passo que elas tiveram quantidades maiores de observações acrescidas.

Empregando o Gerenciador de Experimentos e o Tamanho Ótimo Amostral, foram realizados dois estudos: uma Análise de Variância (ANOVA) Fatorial 4x5 e uma análise empregando a Correlação de Spearman. Inicialmente, a ANOVA Fatorial demonstrou que o desempenho dos alunos foi diretamente influenciado pela interação entre as funcionalidades habilitadas no $\mathrm{CX}$ e a disciplina cursada pelos estudantes. Tal resultado evidencia que apenas o uso de uma ferramenta de apoio ao ensino não deve ser caracterizado como o único fator responsável por elevações significativas no desempenho de estudantes sob análise. É preciso, também, observar a natureza das disciplinas que estão sob investigação, visto que disciplinas que fazem parte de eixos de formação diferentes possuem seus próprios fluxos de apresentação dos conteúdos, compreensão das temáticas e avaliação do processo de aprendizagem.

O estudo que empregou a Correlação de Spearman foi realizado para se averiguar a relação entre o desempenho acadêmico dos estudantes e seus fluxos de interação com o AEU. A partir deste teste, percebeu-se que o nível de utilização do CX apresentou influência sobre a maneira com que os usuários interagiram com a plataforma. Tal indício foi confirmado a partir da análise de que todas as turmas que empregaram o sistema com todos os módulos habilitados apresentaram alguma correlação, ao passo que as amostras que utilizaram o AEU de modo isolado não alcançaram resultados similares.

A disciplina de pós-graduação investigada apresentou uma correlação baixa, o que gerou mais indícios de que o uso de plataformas de apoio ao ensino não se mostra como uma proposta muito relevante para alunos de pós-graduação. Para turmas de graduação, a utilização destas tecnologias se mantém como um tópico de alta relevância para pesquisas científicas, visto que foram observados benefícios significativos para todas as disciplinas analisadas neste nível de ensino. 
VIII Congresso Brasileiro de Informática na Educação (CBIE 2019)

Anais dos Workshops do VIII Congresso Brasileiro de Informática na Educação (WCBIE 2019)

\section{Agradecimentos}

O presente trabalho foi realizado com apoio da Coordenação de Aperfeiçoamento de Pessoal de Nível Superior - Brasil (CAPES) - Código de Financiamento 001. Os autores também agradecem às agências de pesquisa FAPEMIG e CNPq, ao PET/SESu/MEC e ao Instituto Federal do Sul de Minas Gerais (IFSULDEMINAS) pelo apoio concedido.

\section{Referências}

Brant-Ribeiro, T. and Cattelan, R. (2018). Análise de Variância Fatorial do Desempenho de Estudantes sob a Influência de Aprimoramentos Progressivos em Plataformas de Apoio ao Ensino. In Anais do SBIE, volume 1, pages 1233-1242, Fortaleza, BR.

Brant-Ribeiro, T. and Cattelan, R. G. (2016). Determinação de um Tamanho Ótimo Amostral para a Análise do Desempenho de Estudantes em Ambientes Educacionais Ubíquos. RBIE, 24(3):86-96.

Cai, S. (2018). Case studies of augmented reality applications for authentic learning. In Authentic Learning Through Advances in Technologies, pages 115-134. Springer.

Dickson, P. E., Warshow, D. I., Goebel, A. C., Roache, C. C., and Adrion, W. R. (2012). Student Reactions to Classroom Lecture Capture. In Proceedings of the $17^{\text {th }}$ ACM ITiCSE, pages 144-149. ACM.

Efron, B. (1979). Bootstrap Methods: Another Look at the Jackknife. The Annals of Statistics, 7(1):1-26.

Gasevic, D., Mirriahi, N., Dawson, S., and Joksimović, S. (2017). Effects of instructional conditions and experience on the adoption of a learning tool. Computers in Human Behavior, 67:207-220.

Internet World Stats (2018). World Internet User Statistics and World Population Stats, 2018. URL: http: / /www. internetworldstats.com/stats.htm. Acesso em Setembro/2019.

Knight, D. B., Brozina, C., Stauffer, E. M., Frisina, C., and Abel, T. D. (2015). Developing a learning analytics dashboard for undergraduate engineering using participatory design. In 2015 ASEE Annual Conference \& Exposition, pages 1-11, Seattle, Washington. ASEE Conferences.

Lessman, K. J. and Atkins, R. E. (1963). Optimum Plot Size and Relative Efficiency of Lattice Designs for Grain Sorghum Yield Tests. Crop Science, 3(6):477-481.

Meier, V. D. and Lessman, K. J. (1971). Estimation of Optimum Field Plot Shape and Size for Testing Yield in Crambe abyssinica Hochst. Crop Science, 11(5):648-650.

Mouri, K., Ogata, H., and Uosaki, N. (2015). Analysis of ubiquitous learning logs using social network analysis. International Journal of Mobile Learning and Organisation, 9(2):101-123.

Ozer, D. J. (1985). Correlation and the Coefficient of Determination. Psychological Bulletin, 97(2):307-315. 